\title{
A 4.3 W 977 nm ytterbium-doped jacketed-air-clad fiber amplifier
}

\author{
D. B. S. Soh, C. Codemard, J. K. Sahu, J. Nilsson, V. Philippov, C. Alegria, Y. Jeong
}

A cladding pumped jacketed-air-clad ytterbium-doped fiber amplifier operating at $977 \mathbf{n m}$ produced 4.3 W of single-mode output power with $300 \mathrm{~mW}$ of input power from a diode seed laser. The smallsignal gain was $19 \mathrm{~dB}$.

\section{Introduction}

High power sources at $980 \mathrm{~nm}$ such as laser diodes or fiber lasers can be used for pumping of erbium doped fiber devices. If frequency-doubled to $488-490 \mathrm{~nm}$ green-blue light, such sources can also replace bulky and highly inefficient argon ion lasers. Consequently, alternatives such as optically pumped, vertically emitting, semiconductor lasers as well as cladding-pumped ytterbium-doped fiber lasers (YDFLs) have been investigated for high-power 980 $\mathrm{nm}$ operation at the watt-level $[1,2]$ and more recently at the multi-watt level [3]. Q-switching has also been demonstrated [4]. Alternatively, it is possible to make a high-power $980 \mathrm{~nm}$ source using a high power amplifier in a master oscillator - power amplifier (MOPA) configuration. This can bring important advantages in terms of narrow line-width or even single-frequency operation, and improved temporal control for pulsed applications. Also tunability can be readily implemented. Here, we report what we believe is the first cladding-pumped ytterbiumdoped fiber amplifier (YDFA) operating in the $980 \mathrm{~nm}$ wavelength range. An output power of $4.3 \mathrm{~W}$ was reached with a seed power of $300 \mathrm{~mW}$ from a grating-stabilized diode laser.

\section{Amplifier Fiber Design and Experimental Setup}

The YDFA is based on an ytterbium-doped jacketed-air clad (JAC) fiber [1] (inset Fig. 1). The air-clad structure of the fiber increases the numerical aperture of the inner cladding, allowing more pump power to be launched. The small size of the inner cladding $(\sim 25 \mu \mathrm{m})$ enhances the pump absorption. Therefore a strong population inversion is achieved due to an efficient pump absorption. Furthermore, the ring doping technique has been used to ensure that the YDFL works as a two level system (emission at $980 \mathrm{~nm}$ ) rather than as a quasi-four-level system (emission at $1030 \mathrm{~nm}$ ) [5]. Ring-doping reduces the ground-state absorption by reducing the overlap of the signal mode with the Yb-doped gain medium. This promotes the two-level $980 \mathrm{~nm}$ emission relative to the quasi-four-level emission, which is less affected by ground-state absorption.

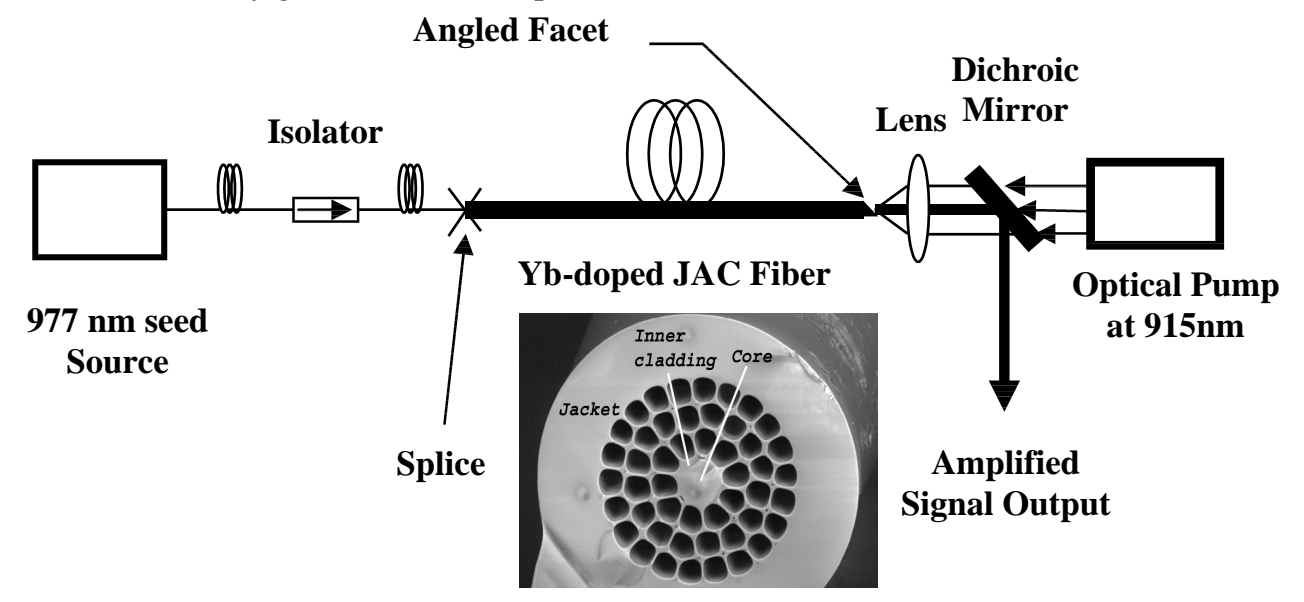

Fig. 1. $977 \mathrm{~nm}$ Yb-doped JAC fiber amplifier experimental setup 
The YDFA experimental set-up is shown in Fig. 1. In the experiments, a $72 \mathrm{~cm}$ long JAC fiber was pumped at 915 $\mathrm{nm}$ by a multi-emitter broad-area laser diode source. The fiber length was optimized for efficient two-level operation. A dichroic mirror was placed between the fiber and the pump in order to extract the amplified signal. The pump was launched into the fiber through a $0.55 \mathrm{NA}, 4.5 \mathrm{~mm}$ focal length collimating lens. The pump power launched into the inner cladding of the JAC fiber was measured to $11 \mathrm{~W}$. The signal output end of the JAC fiber was angle cleaved $(\sim 7 \mathrm{deg})$ in order to suppress back-reflections. In the signal input end, the JAC fiber was spliced to an optical isolator for suppression of feedback and protection of the diode seed laser. Up to $300 \mathrm{~mW}$ of seed power could be launched into the JAC fiber.

\section{Results}

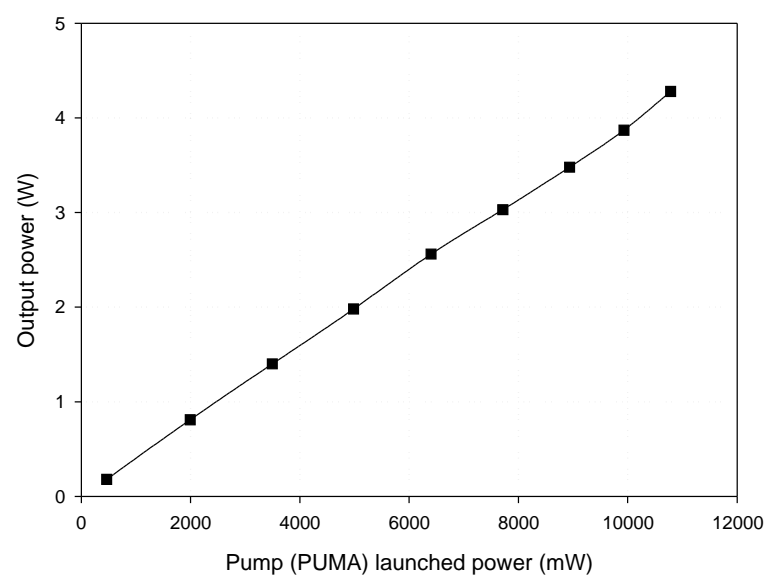

Fig. 2. JAC YDFA output power with respect to launched pump power

Fig. 2 shows the amplified signal output power versus launched $915 \mathrm{~nm}$ pump power. With $11 \mathrm{~W}$ of launched pump power, the output power of the amplifier reached $4.3 \mathrm{~W}$ at $977 \mathrm{~nm}$ for $300 \mathrm{~mW}$ input signal. The gain of the amplifier was then $11.6 \mathrm{~dB}$. The input signal wavelength was $977 \mathrm{~nm}$. Figure 3 shows the amplifier gain with (a) the input signal power fixed at $275 \mathrm{~mW}$, and (b) the pump power fixed at $9 \mathrm{~W}$. The small signal gain was measured to be $19 \mathrm{~dB}$ with $0 \mathrm{dBm}$ seed source.

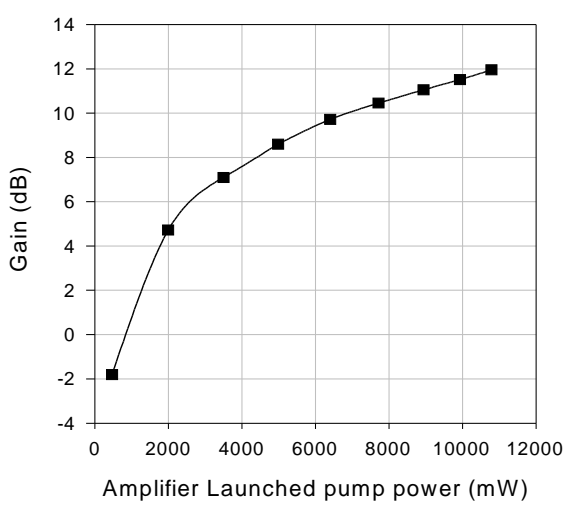

(a)

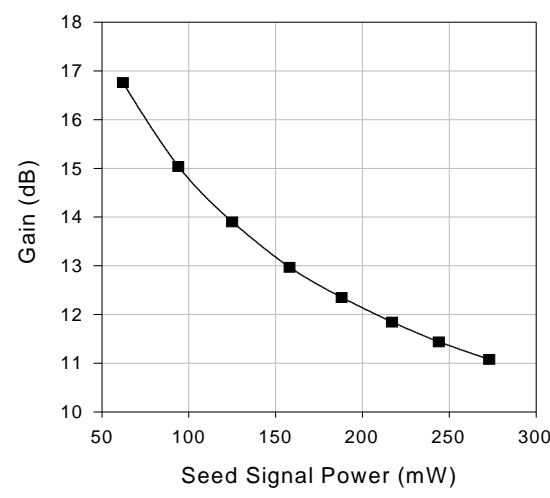

(b)

Fig. 3 JAC YDFA gain characteristics for (a) seed signal power fixed at $275 \mathrm{~mW}$, (b) launched pump power fixed at $9 \mathrm{~W}$.

Fig. 4 shows the typical output spectrum of the JAC YDFA. The linewidth of $977 \mathrm{~nm}$ amplified signal was measured to be $0.2 \mathrm{~nm}$, which was identical to that of the seed source. The output beam quality was measured and an $\mathrm{M}^{2}$ value of 1.14 was found. 


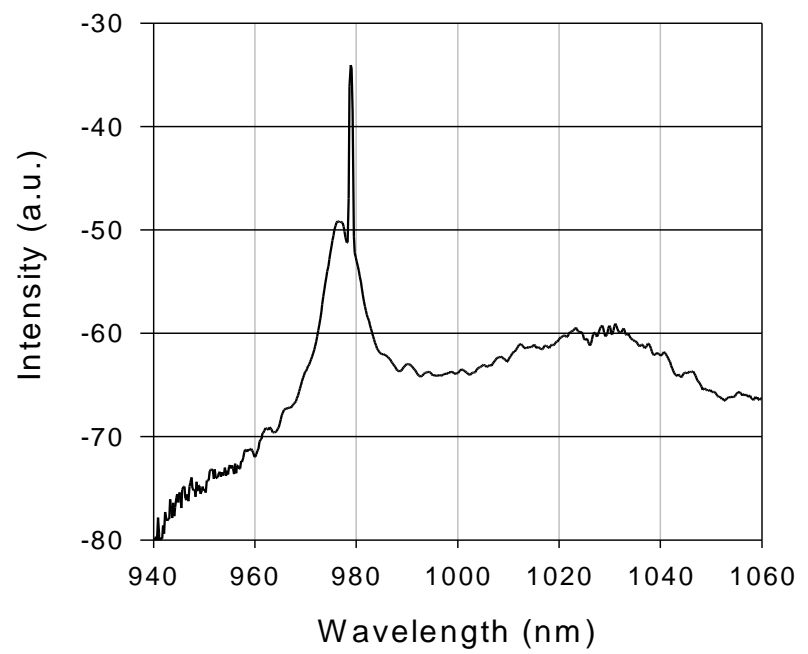

Fig. 4. JAC YDF amplifier output spectrum with $100 \mathrm{~mW} 977 \mathrm{~nm}$ seed signal (OSA resolution $0.5 \mathrm{~nm}$ )

\section{Conclusion}

We have presented a cladding-pumped $977 \mathrm{~nm}$ YDFA based on a ring-doped JAC fiber. The YDFA produced $4.3 \mathrm{~W}$ of single mode output power in a MOPA configuration, when seeded by $300 \mathrm{~mW}$ of power from a laser diode. The system was spliced together so that the signal remained in fiber or fiber-pigtailed components all the way to the output of the JAC YDF. A MOPA with a diode laser and fiber amplifier is a very versatile concept that allows for precise spectral and temporal control.

\section{References}

1. L. B. Fu, R. Selvas, M. Ibsen, J. K. Sahu, S.-U. Alam, J. Nilsson, D. J. Richardson, D. N. Payne, C. Codemard, S. Goncharev, I. Zalevsky, and A. B. Grudinin, "An-8-channel fiber DFB laser WDM transmitter pump with a single $1.2 \mathrm{~W}$ Yb-fibre laser operated at $977 \mathrm{nm",} \mathrm{Technical} \mathrm{digest}$ $28^{\text {th }}$ European Conference on Optical Communication ECOC-2002, Copenhagen, September 2002, 08.3-5

2. L. A. Zenteno, J. D. Minelly, A. Liu, A. J. G. Ellison, S. G. Crigler, D. T. Walton, D. V. Kuksenkov, and M. J. Dejneka, "1 W single transverse-mode Yb-doped double clad fiber laser at $978 \mathrm{~nm}$," Electron. Lett. 37, 819-820 (2001)

3. K. H. Ylä-Jarkko, R. Selvas, D. B. S. Soh, J. K. Sahu, C. A. Codemard, J. Nilsson, S. A. Alam, and A. B. Grudinin, “A 3.5 W 977 nm cladding-pumped jacketed-air clad ytterbium-doped fiber laser", OSA conference on Advanced Solid-State Photonics, San Antonio, February 2003, Postdeadline Paper

4. R. Selvas, J. K. Sahu, and J. Nilsson, "Q-switched 980 nm Yb-doped fiber laser", in OSA Trends in Optics and Photonics (TOPS) Vol. 73 , Conference on Lasers and Electro-Optics, Technical Digest (Optical Society of America, Washington DC, 2002), pp. 565 - 566

5. J. Nilsson, J. D. Minelly, R. Paschotta, D. C. Hanna, and A. C. Tropper, "Ring-doped cladding-pumped single-mode three-level fiber laser", Opt. Lett. 23, 355-357 (1998) 